\title{
Spin-Charge Locking and Tunneling into a Helical Metal
}

\author{
P. $\mathrm{Schwab}^{a}$, R. Raimondi ${ }^{b}$, C. Gorini ${ }^{c}$ \\ ${ }^{a}$ Institut für Physik, Universität Augsburg, 86135 Augsburg, Germany \\ ${ }^{b}$ CNISM and Dipartimento di Fisica "E. Amaldi", Università Roma Tre, 00146 Roma, Italy \\ ${ }^{c}$ Institut de Physique et Chimie des Matériaux de Strasbourg (UMR 7504), \\ CNRS and Université de Strasbourg, 23 rue du Loess, BP 43, F-67034 Strasbourg Cedex 2, France
}

(Dated: March 22, 2022)

\begin{abstract}
We derive a kinetic equation for the electrons moving on the surface of a three-dimensional topological insulator. Due to the helical nature of the excitations backward scattering is suppressed in the collision integral, and the spin dynamics is entirely constrained by that of the charge. We further analyze the tunneling between the helical and a conventional metal or ferromagnet. We find that the tunnel resistance strongly depends on the angle between the magnetization in the ferromagnet and the current in the helical metal. A nonmagnetic layer on top of the helical metal amplifies the current-induced spin polarization.
\end{abstract}

Topological insulators $\$ 122$ have recently attracted considerable interest, especially after their experimental discovery in two ${ }^{3}$ and three dimensions ${ }^{4}-7$. While insulating in the bulk, such materials possess gapless helical edge states whose existence depends on - and is protected by - time reversal invariance ${ }^{8}$ 14. This makes the latter robust against time-reversal symmetric perturbations (such as impurity scattering) and at the same time very sensitive to time-reversal breaking ones (such as magnetic fields). When the topological insulator is a threedimensional system, the gapless excitations are confined to its surface and form a two-dimensional conductor and presents novel and interesting properties, see for example Ref. 15 for a recent summary. In particular, Burkov and Hawthorn $\frac{16}{16}$ considered the problem of spin-charge coupled transport on a helical metal and derived diffusion equations for charge and spin. They predicted a distinctive magnetoresistance effect when the helical metal is placed between a ferromagnet and a normal metal. In this paper we extend their work in several ways. We first derive a kinetic equation which is valid even beyond the diffusive regime. In this latter regime we obtain a diffusion equation which agrees with that of Burkov and Hawthorn as far as the charge component is concerned. On the other hand for the spin density we find a different behaviour, namely the spin dynamics is constrained to follow the charge one. Secondly, we consider the effect of bringing the helical metal in contact with a ferromagnet and discuss its unconventional magnetoresistance.

For the simplest case the effective Hamiltonian describing the surface states of a topological insulator has the form $2 \mid 17$

$$
H=v_{F} \mathbf{k} \times \mathbf{e}_{z} \cdot \boldsymbol{\sigma},
$$

where the parameter $v_{F}$ is the velocity of the gapless excitations, $\mathbf{e}_{z}$ is a unit vector perpendicular to the surface, $\mathbf{k}$ is the two-dimensional momentum operator, $\boldsymbol{\sigma}$ are the Pauli matrices, and units of measure such that $\hbar=1$ have been used. The eigenstates of $H$ form two bands with linear dispersion, $\epsilon_{ \pm}= \pm v_{F} k$, and we will assume in the following that the Fermi energy is located deep enough in the upper band for states in the lower band to remain fully occupied and thus not relevant for the dynamics of the system.

Because of the helical nature of the excitations, such a surface conductor is called a helical metal and presents novel and interesting properties. For instance the velocity operator is given by $\dot{\mathbf{x}}=v_{F} \mathbf{e}_{z} \times \boldsymbol{\sigma}$, so that the particle current becomes $\mathbf{j}=2 v_{F} \mathbf{e}_{z} \times \mathbf{s}$, s being the spin polarization. This means that the particle current is entirely constrained by the spin density or that, vice versa, the in-plane components of the spin density are constrained by the particle current.

Such a constraint will also become apparent later in the kinetic equation for a disordered helical metal. The equation will be valid when the Fermi energy is far from the Dirac point and satisfies the condition $\epsilon_{F} \gg 1 / \tau$, with $\tau$ the scattering time. We will follow a procedure similar to what was done for graphene in Ref. [18. The starting point is the retarded Green function which in the absence of disorder reads

$$
G_{s s^{\prime}}^{R}=G_{0}^{R}\left[\sigma_{0}\right]_{s s^{\prime}}+\mathbf{G}^{R} \cdot[\boldsymbol{\sigma}]_{s s^{\prime}},
$$

where

$$
G_{0}^{R}=\frac{1}{2}\left(G_{+}^{R}+G_{-}^{R}\right)
$$

and

$$
\mathbf{G}^{R}=\frac{1}{2} \hat{\mathbf{k}} \times \mathbf{e}_{z}\left(G_{+}^{R}-G_{-}^{R}\right),
$$

with

$$
G_{ \pm}^{R}=\left(\epsilon \mp v_{F} k+\mu+\mathrm{i} 0^{+}\right)^{-1}
$$

and $\hat{\mathbf{k}}$ being the unit vector in the $\mathbf{k}$-direction. For clarity we included in Eq. (2) the spin indices $s$ and $s^{\prime}$. The Green function $\breve{G}$ has the two-by-two matrix structure of the Keldysh formalism

$$
\check{G}(\mathbf{R}, T ; \mathbf{k}, \epsilon)=\left(\begin{array}{cc}
G^{R}(\mathbf{R}, T ; \mathbf{k}, \epsilon) & G(\mathbf{R}, T ; \mathbf{k}, \epsilon) \\
0 & G^{A}(\mathbf{R}, T ; \mathbf{k}, \epsilon)
\end{array}\right),
$$

where $\mathbf{R}$ and $T$ are the center-of-mass space and time coordinates, while $\mathbf{k}$ and $\epsilon$ are the Fourier transformed 
variables of the relative coordinates. The left-right subtracted Dyson equation reads

$$
\partial_{T} \check{G}+\frac{v_{F}}{2}\left\{\mathbf{e}_{z} \times \boldsymbol{\sigma} \cdot \partial_{\mathbf{R}}, \check{G}\right\}+\mathrm{i}[H, \check{G}]=-\mathrm{i}[\check{\Sigma}, \check{G}],
$$

where [,] and $\{$,$\} are the commutator and anticommu-$ tator. On the right-hand-side of the equation the selfenergy $\check{\Sigma}$ appears. For a delta-correlated impurity potential with $\left\langle V(\mathbf{x}) V\left(\mathbf{x}^{\prime}\right)\right\rangle=u^{2} \delta\left(\mathbf{x}-\mathbf{x}^{\prime}\right)$ and within the Born approximation the self-energy is proportional to the Green function, integrated over the momentum

$$
\check{\Sigma}(\mathbf{R}, T ; \epsilon)=u^{2} \int \frac{d^{2} k}{(2 \pi)^{2}} \check{G}(\mathbf{R}, T ; \mathbf{k}, \epsilon) .
$$

We define a quasiclassical Green function as

$$
\check{g}(\mathbf{R}, T ; \hat{\mathbf{k}}, \epsilon)=\frac{\mathrm{i}}{\pi} \int \mathrm{d} \xi \check{G}(\mathbf{R}, T ; \mathbf{k}, \epsilon),
$$

where $\xi=v_{F} k-\mu$ and the integration is performed in the vicinity of the Fermi energy. From Eqs. (2)-(5), one obtains the retarded component of $\check{g}$ for energies close to the Fermi level $\left(|\epsilon| \ll v_{F} k_{F}\right)$ as

$$
g^{R}=\frac{1}{2}+\frac{1}{2} \hat{\mathbf{k}} \times \mathbf{e}_{z} \cdot \boldsymbol{\sigma},
$$

i.e. $g^{R}$ is a projector on the upper band of the Hamiltonian (1). The self-energy reads

$$
\check{\Sigma}=-\frac{\mathrm{i}}{\tau}\langle\check{g}\rangle
$$

where $1 / \tau=\pi N_{0} u^{2}, N_{0}=k_{F} /\left(2 \pi v_{F}\right)$ is the singleparticle density of states at the Fermi energy and $\langle\check{g}\rangle$ is the average of the quasiclassical Green function over the Fermi surface. Finally, for the Keldysh component of the Green function we find from Eq. 77) the kinetic equation

$$
\begin{gathered}
\partial_{T} g+\frac{v_{F}}{2}\left\{\mathbf{e}_{z} \times \boldsymbol{\sigma} \cdot \partial_{\mathbf{R}}, g\right\}+\mathrm{i} v_{F} k_{F}\left[\hat{\mathbf{k}} \times \mathbf{e}_{z} \cdot \boldsymbol{\sigma}, g\right] \\
=-\frac{1}{\tau} g+\frac{1}{\tau}\langle g\rangle+\frac{1}{2 \tau}\left\{\hat{\mathbf{k}} \times \mathbf{e}_{z} \cdot \boldsymbol{\sigma},\langle g\rangle\right\}
\end{gathered}
$$

Notice that this is a matrix equation in spin space, $g_{s s^{\prime}}=g_{0}\left[\sigma_{0}\right]_{s s^{\prime}}+\mathbf{g} \cdot[\boldsymbol{\sigma}]_{s s^{\prime}}$. However, its structure can be considerably simplified. Equation 12 is derived under the assumption that $v_{F} k_{F}$ is much larger than all other energy scales in the problem. Therefore, the leading contribution to $g$ must commute with $\hat{\mathbf{k}} \times \mathbf{e}_{z} \cdot \boldsymbol{\sigma}$, or in other words, $g$ is diagonal in the eigenstates of $H$. Since the lower band has no density of states at the Fermi level, only the upper band contributes to $g$ which is then proportional to the upper-band projector, i.e.

$$
g_{s s^{\prime}}=g_{0}\left(\left[\sigma_{0}\right]_{s s^{\prime}}+\hat{\mathbf{k}} \times \mathbf{e}_{z} \cdot[\boldsymbol{\sigma}]_{s s^{\prime}}\right) .
$$

From the spin-trace of Eq. 12 we obtain then a kinetic equation for $g_{0}$,

$\partial_{T} g_{0}+v_{F} \hat{\mathbf{k}} \cdot \partial_{\mathbf{R}} g_{0}=-\int \frac{d \varphi^{\prime}}{2 \pi} W\left(\varphi-\varphi^{\prime}\right)\left[g_{0}(\varphi)-g_{0}\left(\varphi^{\prime}\right)\right]$, which is just the standard kinetic equation for a metal with an angle dependent scattering potential ${ }^{19 / 20}$. Here, starting from a short range potential, we find

$$
W\left(\varphi, \varphi^{\prime}\right)=\frac{1}{\tau}\left(1+\hat{\mathbf{k}} \cdot \hat{\mathbf{k}}^{\prime}\right)=\frac{1}{\tau}\left(1+\cos \left(\varphi-\varphi^{\prime}\right)\right)
$$

where the cosine term accounts for the absence of backscattering. The spin-dependent contributions to $g$ can be reconstructed from $g_{0}$, cf. Eq. 113):

$$
g_{x} \approx \hat{k}_{y} g_{0}, \text { and } g_{y} \approx-\hat{k}_{x} g_{0}
$$

The $g_{z}$ component is nonzero only to subleading order in $1 / v_{F} k_{F}$ and is after some algebra determined as

$g_{z} \approx \frac{1}{v_{F} k_{F}}\left(\frac{\hat{k}_{x}}{\tau}\left\langle\hat{k}_{y} g_{0}\right\rangle-\frac{\hat{k}_{y}}{\tau}\left\langle\hat{k}_{x} g_{0}\right\rangle+v_{F} \hat{k}_{y} \partial_{x} g_{0}-v_{F} \hat{k}_{x} \partial_{y} g_{0}\right)$.

Equations (14), 16 and (17) are one of the main results obtained in this paper.

It is instructive to study the angular average of the kinetic equation, since the latter is closely related to the continuity equation for the observables. Recall, for example, that the particle and spin densities are obtained from the quasiclassical Green function by taking the angle average and integrating over the energy ${ }^{19}$,

$$
n=-\frac{N_{0}}{2} \int \mathrm{d} \epsilon\left\langle g_{0}\right\rangle+N_{0} e \phi, \mathbf{s}=-\frac{N_{0}}{4} \int \mathrm{d} \epsilon\langle\mathbf{g}\rangle,
$$

where $\phi$ is the scalar electrical potential. From Eq. 14 we obtain the continuity equation for the density as

$$
\partial_{T}\left\langle g_{0}\right\rangle+\partial_{\mathbf{R}} \cdot\left\langle v_{F} \hat{\mathbf{k}} g_{0}\right\rangle=0
$$

Using Eq. 16 we find $v_{F}\left\langle\hat{k}_{x} g_{0}\right\rangle=-v_{F}\left\langle g_{y}\right\rangle$ and $v_{F}\left\langle\hat{k}_{y} g_{0}\right\rangle=v_{F}\left\langle g_{x}\right\rangle$, i.e. we verify the general relations between the particle current and the in-plane spin density stated already below Eq. (1). For the spin density we find

$$
\begin{aligned}
& \partial_{T} s_{x}+\frac{v_{F}}{4} \partial_{y} n+\frac{1}{2 \tau} s_{x}=0 \\
& \partial_{T} s_{y}-\frac{v_{F}}{4} \partial_{x} n+\frac{1}{2 \tau} s_{y}=0
\end{aligned}
$$

from which we identify $2 \tau$ as the spin relaxation time. In (20) and 21) we ignored the scalar electric potential, $\phi$.

In order to compare with Ref. [16] we will now discuss the diffusive limit. The particle density obeys the diffusion equation

$$
\partial_{T} n-D \partial_{\mathbf{R}}^{2} n=0, D=\frac{v_{F}^{2}}{2} \tau_{t r}, \tau_{t r}=2 \tau,
$$

i.e., the particle current is $\mathbf{j}=-D \partial_{\mathbf{R}} n$. The transport time $\tau_{t r}$ being twice as long as the scattering time $\tau$ stems from the absence of backscattering in the helical metal. Equation (22) is consistent with Ref. [16, notice however the different definition of the diffusion constant. 
Since the spin relaxation time is very short (it equals the transport scattering time) the spin dynamics is not diffusive. However, Eqs. (20) and (21) are still valid. In the diffusive limit the time derivative of the spin-density is small compared to the spin relaxation term, i.e. the spin density is given by the spatial derivative of the charge density so again we identify the general relation between particle current and spin density.

We will now analyze the transport through the helical metal when it is contacted via a tunnel junction to a ferromagnet. We will find an unconventional magnetoresistance effect that arises since the tunneling probability between the helical metal and the ferromagnet is strongly angle dependent: the overlap of two spinors with polarization in $\hat{\mathbf{m}}$ - and $\hat{\mathbf{m}}^{\prime}$-directions depends on the angle between the two vectors, $\left|\left\langle\hat{\mathbf{m}} \mid \hat{\mathbf{m}}^{\prime}\right\rangle\right|^{2}=\frac{1}{2}+\frac{1}{2} \hat{\mathbf{m}} \cdot \hat{\mathbf{m}}^{\prime}$. The states in the conduction band of the helical metal are polarized as $\hat{\mathbf{m}}^{\prime}=\hat{\mathbf{k}} \times \mathbf{e}_{z}$, so that the tunneling probability from a state $\hat{\mathbf{m}}$ in the ferromagnet into a state in the helical metal with momentum $\mathbf{k}$ depends on the angle between $\hat{\mathbf{m}}$ and $\mathbf{k}$.

In order to make these considerations more formal we introduce the tunneling Hamiltonian

$$
H_{t}=\sum_{s} \int d^{2} x t(\mathbf{x}) \psi_{s}^{\dagger}(\mathbf{x}) \psi_{F, s}(\mathbf{x})+c . c .
$$

where $\psi_{s}^{\dagger}(\mathbf{x})$ is the field operator for an electron with spin $s$ in the helical metal and $\psi_{F, s}(\mathbf{x})$ that for the ferromagnet. For a point-like (on the quasiclassical scale) contact at $\mathbf{x}=0$ the tunneling amplitude is

$$
t(\mathbf{x})=t \delta(\mathbf{x})
$$

Eq. (23) leads then to an additional contribution to the self-energy of the form

$$
\Sigma_{t}(\mathbf{R}, T ; \epsilon)=|t|^{2} \delta(\mathbf{R}) \int \frac{d^{2} k}{(2 \pi)^{2}} \check{G}_{F}(\mathbf{R}, T ; \mathbf{k}, \epsilon)
$$

where $\check{G}_{F}$ is the Green function of the ferromagnet. We assume that the conduction electrons in the ferromagnet can be described in terms of an incoherent superposition of majority and minority carriers, so we write

$$
\check{G}_{F}=\check{G}_{F, \uparrow} P_{\uparrow}+\check{G}_{F, \downarrow} P_{\downarrow},
$$

where $P_{\uparrow, \downarrow}=\left(\sigma_{0} \pm \hat{\mathbf{m}} \cdot \boldsymbol{\sigma}\right) / 2$ projects on states parallel antiparallel to $\hat{\mathbf{m}}$. The kinetic equation (14) becomes

$$
\begin{gathered}
\left(\partial_{T}+v_{F} \hat{\mathbf{k}} \cdot \partial_{\mathbf{R}}\right) g_{0}=-\frac{1}{\tau} g_{0}+\frac{1}{\tau}\left(\left\langle g_{0}\right\rangle+\hat{\mathbf{k}} \cdot\left\langle\hat{\mathbf{k}}^{\prime} g_{0}\right\rangle\right) \\
-\pi|t|^{2} \delta(\mathbf{R}) N_{\uparrow}\left(1+\hat{\mathbf{m}} \cdot \hat{\mathbf{k}} \times \hat{\mathbf{e}}_{z}\right)\left(g_{0}-\frac{1}{2}\left\langle g_{\uparrow}\right\rangle\right) \\
-\pi|t|^{2} \delta(\mathbf{R}) N_{\downarrow}\left(1-\hat{\mathbf{m}} \cdot \hat{\mathbf{k}} \times \hat{\mathbf{e}}_{z}\right)\left(g_{0}-\frac{1}{2}\left\langle g_{\downarrow}\right\rangle\right), \quad
\end{gathered}
$$

where $g_{\uparrow, \downarrow}$ and $N_{\uparrow, \downarrow}$ are the quasiclassical Green function and the density of states in the ferromagnet. The terms in the second and third line of this equation describe

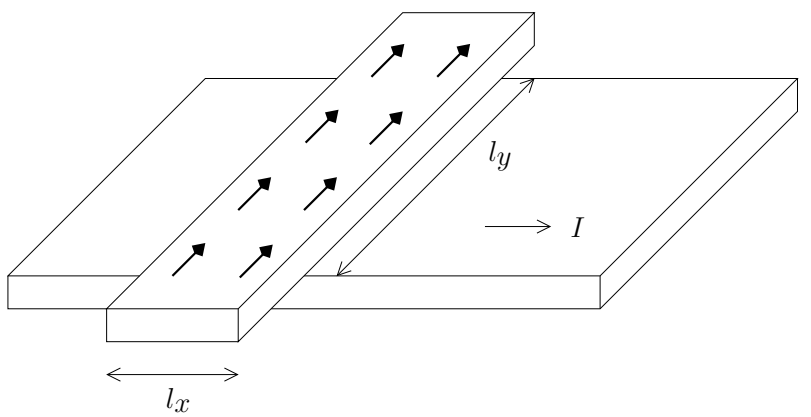

FIG. 1. Schematic view of system under consideration: electrons can tunnel between a ferromagnet (top) and a helical metal (bottom). The size of the contact is $l_{x}$ times $l_{y}$, the current is assumed to flow only in $x$-direction.

tunneling between the helical metal and the spin up or down band of the ferromagnet. As anticipated above, the tunneling probability between the helical metal and the ferromagnet is a $\hat{\mathbf{k}}$-dependent function. After the angular average and integrating over the energy we obtain the following continuity equation for the charge density, $\rho=$ $(-e) n$,

$$
\begin{aligned}
\partial_{T} \rho+\partial_{\mathbf{R}} \cdot \mathbf{j} & =-\delta(\mathbf{R})\left[G_{\uparrow}\left(U-U_{\uparrow}\right)+G_{\downarrow}\left(U-U_{\downarrow}\right)\right] \\
& -\delta(\mathbf{R}) \frac{G_{\uparrow}-G_{\downarrow}}{e^{2} N_{0} v_{F}} \hat{\mathbf{e}}_{z} \times \hat{\mathbf{m}} \cdot \mathbf{j}
\end{aligned}
$$

having introduced the tunneling conductances

$$
G_{\uparrow, \downarrow}=\pi e^{2}|t|^{2} N_{0} N_{\uparrow, \downarrow}
$$

and the integrals

$$
U=\frac{1}{2 e} \int \mathrm{d} \epsilon\left\langle g_{0}\right\rangle, U_{\uparrow, \downarrow}=\frac{1}{4 e} \int \mathrm{d} \epsilon\left\langle g_{\uparrow \downarrow}\right\rangle .
$$

The latter have the meaning of a voltage, or more precisely of a magneto-electrochemical potential21, cf. Eq. (18). Sources and sinks for the charge density in the helical metal appear on the right hand side of the equation due to tunneling. One observes that a tunnel current cannot only be driven by a voltage across the contact, but also by a current bias in the helical metal.

For simplicity we will assume in the following equal magneto-electrochemical potentials for spin up and down electrons in the ferromagnet, $U_{\uparrow \downarrow}=U_{\uparrow}=U_{\downarrow}$. Furthermore, instead of a point-like tunnel contact we will consider an extented tunneling region as depicted in Fig. 1. This is achieved by replacing the $\delta$-function on the right hand side of Eq. 28) by an appropriate function characterising the shape of the contact. In particular, we will study in detail a junction with a long extension $l_{y}$ in $y$ direction ${ }^{22}$. We replace then the $\delta$-function in Eq. 28) by

$$
\delta(\mathbf{R}) \rightarrow\left\{\begin{array}{cl}
l_{y}^{-1} \delta(x) & \text { for }-l_{y} / 2<y<l_{y} / 2 \\
0 & \text { otherwise }
\end{array}\right.
$$


and we assume that current flows only in $x$-direction. The continuity equation thus becomes one-dimensional. Due to the remaining one-dimensional $\delta$-function the current density jumps at $x=0$. The size of this jump is determined by integrating the continuity equation with respect to the $x$-coordinate for a region close to the tunneling contact with the result

$j_{+}-j_{-}=-\frac{G_{\uparrow}+G_{\downarrow}}{l_{y}}\left(U-U_{\uparrow \downarrow}\right)+\frac{G_{\uparrow}-G_{\downarrow}}{l_{y} N_{0} v_{F} e^{2}} \frac{j_{+}+j_{-}}{2} \hat{m}_{y}$,

where $j_{ \pm}=j(x= \pm 0)$ is the current right and left from the tunnel contact and we assumed that $\delta(x) j(x)=$ $\delta(x)\left(j_{+}+j_{-}\right) / 2$. When we fix the current left to the contact to zero we determine the tunnel resistance as

$$
R_{t}=\frac{1}{G_{\uparrow}+G_{\downarrow}}-\frac{\hbar}{e^{2}} \frac{\lambda_{F} \hat{m}_{y}}{2 l_{y}} \frac{G_{\uparrow}-G_{\downarrow}}{G_{\uparrow}+G_{\downarrow}},
$$

where for clarity we put back $\hbar$. Through $\hat{m}_{y}$ the tunnel resistance depends on the orientation of the ferromagnet with respect the the $x$-axis, similar to what was found by Burkov and Hawthorn 16 . The relative size of the magnetoresistance effect increases with increasing tunnel conductance being controlled by the dimensionless parameters $\left(G_{\uparrow}-G_{\downarrow}\right) /\left(e^{2} / \hbar\right)$ and $\lambda_{F} / l_{y}$. Apparently the tunnel resistance may even become negative for large enough values of these two parameters. However, as we will see below, when this happens equation (33) is no longer valid and a more careful treatment which takes into account a finite contact area of the tunnel junction is needed. To analyze a junction with a finite width $l_{y}$ and length $l_{x}$ we make the following replacement in Eq. (28): $\delta(\mathbf{R}) \rightarrow\left(l_{x} l_{y}\right)^{-1}$ when $\mathbf{R}$ is inside the tunnel junction and $\delta(\mathbf{R}) \rightarrow 0$ otherwise. This leads to the tunnel resistance

$$
R_{t}=\frac{1}{G_{\uparrow}+G_{\downarrow}} f\left(\frac{\lambda_{F}}{l_{y}} \frac{G_{\uparrow}-G_{\downarrow}}{e^{2} / \hbar} \hat{m}_{y}\right)
$$

with the function $f(x)=x /\left(e^{x}-1\right)$. For small $x, f(x) \approx$ $1-x / 2$ and one recovers Eq. (33). When $x$ increases and becomes of order one, the resistance remains positive, but depends strongly on the sign of $x$, i.e., the orientation of the ferromagnet. For $x>0$, the resistance decreases exponentially in the parameter $\left(G_{\uparrow}-G_{\downarrow}\right) /\left(e^{2} / \hbar\right)\left(\lambda_{F} / l_{y}\right)$, while it increases linearly for $x<0$. The tunnel junction is then acting as a spin-diode.

In this final part of the paper we consider tunneling from the helical metal into a normal, nonmagnetic metal. The tunnel resistance for the charge becomes $R_{t}=1 /\left(G_{\uparrow}+G_{\downarrow}\right)$ since spin up and down have both the same density of states, $N_{\uparrow}=N_{\downarrow}=N_{\uparrow \downarrow}$ and also identical tunnel conductance. Nevertheless tunneling into a normal metal is of interest since, as we will demonstrate, the helical metal injects spin into the normal metal. Going through the same steps as in Eqs. (25) $-(28)$ but now for the spin-density in the normal metal we arrive at

$$
\begin{aligned}
\partial_{t} s^{a}+\partial_{\mathbf{R}} \cdot \mathbf{j}^{a}= & -\frac{\hbar / 2 e^{2}}{R_{t}} \frac{1}{N_{\uparrow \downarrow}} \delta(\mathbf{R}) s^{a} \\
& -\frac{\hbar / 2 e^{2}}{R_{t}} \frac{\delta(\mathbf{R})}{e N_{0} v_{F}}\left(\mathbf{j} \times \mathbf{e}_{z}\right)_{a},
\end{aligned}
$$

where $\mathbf{j}^{a}$ is the spin current in the normal metal and $\mathbf{j}$ the charge current in the helical metal. Whereas the tunneling of charge is controlled by the voltage across the tunnel junction, the tunneling of spin is controlled by the current density in the helical metal, i.e. a current flowing parallel to the junction. Thus injection of a pure spin current into the normal metal is possible. Furthermore, if spin tunneling in and out are balanced so that there is no spin current injection, there is a steady state determined by

$$
\frac{1}{N_{\uparrow \downarrow}} \mathbf{s}=-\frac{1}{e N_{0} v_{F}} \mathbf{j} \times \mathbf{e}_{z},
$$

which is equivalent to

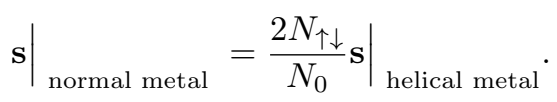

A normal metal on top of the helical metal thus amplifies the current-induced spin polarization.

We finally turn to the question of how robust the effects found are, having in mind that the effective Hamiltonian (1) is only valid in the vicinity of the Dirac point, whereas the interpretation of the experimentally observed Fermi surfaces ${ }^{4 / 577}$ requires at finite doping terms that are quadratic and even cubic in $\mathbf{k}^{23}$. For example, to third order in $\mathbf{k}$, the effective Hamiltonian for the surface states of the topological insulators $\mathrm{Bi}_{2} \mathrm{Te}_{3}$ and $\mathrm{Bi}_{2} \mathrm{Se}_{2}$ is ${ }^{23}$

$$
H=v \mathbf{k} \times \mathbf{e}_{z} \cdot \boldsymbol{\sigma}+\frac{k^{2}}{2 m^{*}}+\frac{\lambda}{2}\left(k_{+}^{3}+k_{-}^{3}\right) \sigma_{z},
$$

where the Dirac velocity contains a second order correction, $v=v_{F}\left(1+\alpha k^{2}\right), k_{ \pm}=k_{x} \pm i k_{y}$, and $\alpha, m^{*}, \lambda$ are the parameters characterizing the strength of the higher order corrections. In order to understand the effect these extra terms in the Hamiltonian have on the magnetoresistance and the spin injection, recall that the origin of both is the helicity of the conduction electrons. The quadratic term in the Hamiltonian (38) has no spin structure, does not affect the helicity of the eigenstates, and therefore cannot qualitatively change our results. The cubic term on the other hand disturbs the helicity of the eigenstates (the angle between the velocity and the spin now depends on the position on the Fermi surface), so for a strong cubic term we leave the region where our results are reliable.

In conclusion we have derived a kinetic equation for a helical model and have shown that the spin dynamics is constrained to follow that of the charge. In the diffusive regime for charge, the spin density is described in terms of the charge density gradient. Furthermore, when the 
helical metal is placed in contact with a ferromagnetic metal, the tunneling current depends on the relative orientation between the current and the polarization in the ferromagnet. In the limit of large tunneling conductance, the device acts as a spin-diode. A helical metal in contact with a normal metal injects spin into the latter, with a rate that can be controlled independently from the injection of charge carriers.

We thank the Deutsche Forschungsgemeinschaft (SPP1285) and the French Agence Nationale de la Recherche (grant no. ANR-08-BLAN-0030-02) for financial support.
1 J. E. Moore, Nat. Phys., 5, 378 (2009).

2 M. Hasan and C. Kane, Rev. Mod. Phys., 82, 3045 (2010).

3 M. Konig, S. Wiedmann, C. Brune, A. Roth, H. Buhmann, L. W. Molenkamp, and S.-C. Qi, X.-Land Zhang, Science, 766 (2007).

4 D. Hsieh, Y. Xia, D. Qian, L. Wray, J. H. Dill, F. Meier, J. Osterwalder, L. Patthey, J. G. Checkelsky, N. P. Ong, A. V. Fedorov, H. Lin, A. Bansil, D. Grauer, Y. S. Hor, R. J. Cava, and M. Z. Hasan, Nature, 460, 1101 (2009).

5 Y. L. Chen, J. G. Analytis, J.-H. Chu, Z. K. Liu, S.-K. Mo, X. L. Qi, H. J. Zhang, D. H. Lu, X. Dai, Z. Fang, S. C. Zhang, I. R. Fisher, Z. Hussain, and Z.-X. Shen, Science, 325, 178 (2009).

6 Y. Xia, D. Qian, D. Hsieh, L. Wray, A. Pal, H. Lin, A. Bansil, D. Grauer, Y. S. Hor, R. J. Cava, and M. Z. Hasan, Nature Physics, 5, 398 (2009).

7 K. Kuroda, M. Arita, K. Miyamoto, M. Ye, J. Jang, A. Kimura, E. E. Krasovskii, E. V. Chulkov, H. Iwasawa, T. Okuda, K. Shimada, Y. Ueda, H. Namatame, and M. Taniguchi, Phys. Rev. Lett., 105, 076802 (2010).

8 C. L. Kane and E. J. Mele, Phys. Rev. Lett., 95, 226801 (2005).

9 C. L. Kane and E. J. Mele, Phys. Rev. Lett., 95, 146802 (2005)
10 B. A. Bernevig, T. L. Hughes, and S.-C. Zhang, Science, 1757 (2006).

11 J. E. Moore and L. Balents, Phys. Rev. B, 75, 121306 (2007)

${ }^{12}$ L. Fu, C. L. Kane, and E. J. Mele, Phys. Rev. Lett., 98, 106803 (2007).

13 S. Murakami, New Journal of Physics, 9, 356 (2007).

14 R. Roy, Phys. Rev. B, 79, 195322 (2009).

15 D. Culcer, E. H. Hwang, T. D. Stanescu, and S. D. Sarma, Phys. Rev. B, 82, 155457 (2010).

16 A. A. Burkov and D. G. Hawthorn, Phys. Rev. Lett., 105, $066802(2010)$

${ }^{17}$ H. Zhang, C.-X. Liu, X.-L. Qui, X. Dai, Z. Fang, and S.-C. Zhang, Nature Physics, 5, 483 (2009).

18 K. Kechedzhi, O. Kashuba, and V. I. Fal'ko, Phys. Rev. B, 77, 193403 (2008).

I9 J. Rammer and H. Smith, Rev. Mod. Phys., 58, 323 (1986)

20 P. Schwab and R. Raimondi, Ann. Phys. (Berlin), 12, 471 (2003).

21 M. Johnson and R. H. Silsbee, Phys. Rev. B, 35, 4959 (1987).

22 Our equations apply to wide contacts provided they can be modeled as a series of incoherent tunnel junctions.

${ }^{23}$ L. Fu, Phys. Rev. Lett., 103, 266801 (2010). 